\title{
Marcadores de estrés musculoesquelético en los huesos largos de una población española (Wamba, Valladolid)
}

\author{
IMMACULADA LÓPEZ-BUEIS \\ Director: \\ Dr. Gonzalo J. Trancho, \\ Profesor Titular de Antropología \\ Facultad de Biología de la Universidad Complutense de Madrid.
}

\section{Introducción}

El estudio de los restos óseos humanos resulta de gran importancia para el conocimiento de la forma de vida de las poblaciones. El esqueleto es sensible al medio que le rodea, de modo que factores tales como la dieta, las enfermedades, e incluso la actividad física realizada por el individuo, pueden dejar huellas, incluyendo variantes normales y patológicas, sobre el tejido óseo. De este modo, el análisis del esqueleto puede proporcionar información sobre la interacción entre el medio ambiente y el comportamiento del individuo o su forma de vida.

De acuerdo con Robb (1994, 1998), las impresiones musculares aparecen debido a la función del esqueleto como un sistema de palancas. Biológicamente, las marcas musculares parecen ser el resultado de una acumulación de microtraumas en los puntos de origen e inserción muscular, por lo que su expresión ósea es bastante compleja. Por ejemplo, una tuberosidad deltoidea visualmente prominente puede estar reflejando tanto la musculatura robusta que soporta, como la gracilidad relativa del hueso subyacente, y la prominencia de la tuberosidad frecuentemente no se corresponde con la rugosidad de las marcas musculares encontradas sobre su superficie (Steen y Lane, 1998). Estas impresiones además, pueden estar influidas por traumas o enfermedades metabólicas, y el factor más importante a considerar, aparte de la actividad, es la edad del individuo (Shaibani et al., 1993; Rogers y Waldron, 1990).

El estado actual de conocimiento sugiere que las impresiones musculares pueden usarse para interpretar actividades siempre que se controle la edad del individuo. Por otro lado, resulta importante distinguir las entesopatías mecánicas de las de origen inflamatorio o metabólico, debido a que la aparición de dichas entesopatías puede estar relacionada con diversos procesos patológicos, tales como la hiperostósis esquelética difusa idiopática (DISH), la psoriasis, la enfermedad de Reiter, la artritis reumatoide, etc. Las observaciones realizadas en el campo de la medicina del deporte u ocupacional indican que algunas entesopatías mecánicas están correlacionadas con actividades específicas. En ciertos casos, podrían ser útiles para reconstruir ciertas actividades de poblaciones antiguas teniendo cuidadosamente en cuenta el contexto arqueológico e histórico (Pálfi y Dutour, 1996).

Dentro del estudio de los marcadores de actividad física creemos que es importante mencionar algunos aspectos que pueden limitar su aplicación. Dados los estrechos márgenes de variación que puede sufrir el hueso frente a condiciones de presión ambiental, o se forma nuevo hueso o se destruye el previamente existente, y debido a la participación de los mismos músculos en distintas actividades, es muy posible que el desarrollo de las inserciones musculares pueda ser debido a un amplio rango de actividades. Además, hay que tener en cuenta la variación individual, genética (Churchill y Morris, 1998), es decir, la realización de una actividad no tiene por qué afectar de la misma manera a todos los individuos que la realicen. Otro factor que en nuestra opinión tiene gran importancia, es la cronicidad con que se lleve a cabo una actividad, es decir, cuanto tiempo y/o durante cuantos años se haya dedicado un individuo a la realización de una actividad en concreto. Sin embargo, el análisis de las frecuencias observadas, así como su diferente distribución por lado y sexo, nos puede informar 
acerca de patrones generales de actividad o condiciones de presión ambiental a las que estuvo sometida la población.

En este trabajo nos propusimos estudiar diferentes marcadores óseos para intentar encontrar patrones que indiquen qué tipo de actividad realizaban los individuos de la población analizada y hasta qué grado estos marcadores pueden contribuir al estudio del dimorfismo sexual que presenta la colección.

La serie estudiada procede de un osario situado en el Monasterio de Santa María, refundado en el año 928 y en el que en el siglo XII se asentaron los Caballeros Hospitalarios de la Orden de San Juan de Jerusalén (Rucquoi, 1987). El límite cronológico superior del osario se sitúa en el siglo XVII, cuando la necesidad de espacio hizo que se vaciaran las tumbas antiguas, cuyos restos pasaron a formar parte del osario; mientras que como límite inferior hemos considerado el siglo $\mathrm{XV}$, que es el periodo durante el cual se llevaron a cabo numerosas ampliaciones arquitectónicas que pudieron promover la remoción y limpieza de tumbas, contribuyendo así a la ampliación del museo-osario, y además es en este momento cuando se recogen las primeras informaciones acerca del origen del osario.

Los antecedentes históricos de los que disponemos nos muestran un pueblo eminentemente agricultor, con influencia de la ganadería. Estos datos se corresponden con los resultados obtenidos en un estudio de la dieta de diversas poblaciones de la Península Ibérica, entre las que se incluía Wamba (Pérez-Pérez et al., 1991) y donde se indicaba que la actividad económica preferencial de esta colección estaba probablemente relacionada con la agricultura. También se apuntaba un mayor consumo cárnico en la muestra masculina respecto a la femenina, existiendo dimorfismo sexual significativo. Este resultado vendría apoyado por documentación histórica que pone de manifiesto la existencia de una actividad ganadera en aquella villa, que pudo llegar a ser importante, pero siempre por debajo de la actividad agrícola.

En general podemos situar Wamba en un contexto de aldea medieval, de economía predominantemente agrícola, y no muy poblada (Martín, 1986). Podemos establecer a priori que esta población estaba sometida a una fuerte presión ambiental, debido a la importante actividad física realizada desde muy temprana edad, puesto que los niños se incorporaban al trabajo en el campo por ley a los 12 años, aunque sabemos que ya desde antes realizaban tareas complementarias junto a los ancianos, como en el espigado de los campos una vez segados (Martín, 1986). Además esperaríamos encontrar un dimorfismo sexual acusado, debido a la existencia de una división del trabajo entre ambos sexos, puesto que mientras el varón era el principal encargado de las tareas agrícolas, la mujer llevaba la casa, las aves del corral, la huerta, y ayudaba a su marido en época de la cosecha.

\section{Material y Metodos}

La muestra que ha servido para la realización de este trabajo proviene del osario del Monasterio de Santa María de Wamba, Valladolid, datado entre los siglos XV y XVII. Consta de 516 húmeros, 186 radios, 198 cúbitos, 671 fémures y 561 tibias.

Se analizaron por separado los huesos de ambos lados, con el fin de estudiar si existían diferencias significativas en las variables cuantitativas y en el análisis de los caracteres epigenéticos, marcadores de actividad física y patologías. Sólo se analizaron los huesos adultos, considerándose como tal aquellos que presentaban ambas epífisis totalmente fusionadas.

La estimación del sexo se realizó utilizando criterios cualitativos. Se incluyó el aspecto general de hueso, su robustez o esbeltez, y el grado de desarrollo de las zonas de origen e inserción muscular. También se tuvo muy en cuenta el desarrollo y la forma de las epífisis. Solamente se sexaron los huesos largos clasificados como adultos.

Se codificó la presencia de alteraciones a nivel de inserción muscular de los huesos largos siguiendo un criterio de Ausencia-Presencia. Todos los caracteres considerados aparecen descritos en Kennedy (1989) y aparecen en la Tabla 1.

\section{Resultados y Discusión}

Debido a la existencia de una serie de limitaciones previas en esta población, como son la falta de correspondencia entre lados y entre las diferentes estructuras anatómicas, y/o la imposibilidad de dividir la muestra por intervalos de edad, resulta imposible identificar actividades manuales como las desarrolladas por profesionales que seguramente existían en la población, por ejemplo, herreros, molineros, etc. Sin embargo, sí que podemos estudiar la frecuencia de los marcadores de actividad considerados, así como la presencia de asimetrías o dimorfismo sexual, lo 
Tabla 1: Marcadores de estrés musculoesquelético considerados en este trabajo.

\begin{tabular}{||l||l||}
\hline \hline HÚMERO & Aplastamiento de la tuberosidad mayor. \\
\hline \hline & Aplastamiento de la tuberosidad menor. \\
\hline & Surco en la inserción del músculo pectoral. \\
\hline & Surco en la inserción del músculo redondo mayor. \\
\hline & Exóstosis en la epitróclea. \\
\hline \hline RADIO & Exóstosis en el epicóndilo. \\
\hline \hline CÚBITO & Exóstosis en la tuberosidad bicipital. \\
\hline & Desarrollo de la cresta del músculo supinador. \\
\hline & Exóstosis en el olécranon. \\
\hline & Desarrollo de la cresta del músculo pronador. \\
\hline & Presencia de un surco asociado a la inserción del músculo pronador. \\
\hline FÉMUR & Exóstosis en el trocánter mayor. \\
\hline & Exóstosis en el trocánter menor. \\
\hline & Exóstosis en la tuberosidad glútea. \\
\hline & Exóstosis en la cresta anterior intertrocantérica. \\
\hline & Exóstosis en la línea áspera. \\
\hline & Depresión y exóstosis en la cara posterior del cóndilo medial. \\
\hline \hline TIBIA & Exóstosis en la tuberosidad tibial. \\
\hline & Exóstosis en la línea del sóleo. \\
\hline & Exóstosis en la inserción del músculo flexor de los dedos. \\
\hline
\end{tabular}

cual puede estar indicando cronicidad en la realización de alguna actividad, así como diferencias en las tareas realizadas por ambos sexos.

En la Tabla 2 aparecen los resultados acerca de las entesopatías encontradas en el miembro superior. En relación al húmero, se encontró asimetría en la presencia de un surco a nivel de la inserción del músculo pectoral en varones $\left(\mathrm{X}^{2} 1=\right.$ $5.56, \mathrm{p} \leq 0.05)$ y en el aplastamiento de la tuberosidad mayor en los húmeros femeninos $\left(\mathrm{X}^{2} 1\right.$ $=4.08, \mathrm{p} \leq 0.05)$ siendo más frecuentes en el lado derecho. La parte superior de la tuberosidad mayor es el punto de inserción de los músculos supra e infra espinosos. El músculo supraespinoso es responsable de la abducción del brazo (Kapandji, 1996 a) y es relativamente frecuente que se produzca la alteración del tendón de este músculo por sobrecarga o traumatismo (Platzer, 1987). El músculo infraespinoso refuerza la cápsula de la articulación del hombro y su función principal es rotar lateralmente el brazo (Platzer, 1987). La presencia de asimetría a este nivel ya ha sido descrita en otros trabajos (Stirland, 1993) y se ha relacionado con un soporte asimétrico del peso durante una actividad habitual o repetida. El hecho de que se produzca asimetría en mujeres, puede deberse a la realización de una actividad que exigiera únicamente la participación del brazo derecho, como podría ser, por ejemplo, llevar pesos sobre ese hombro al ir a por agua o llevar la ropa a lavar, etc., aunque la pequeña frecuencia de este marcador en la población, un $11.5 \%$, no parece estar indicando la realización de una actividad mayoritaria.

Respecto al dimorfismo sexual, se obtuvieron diferencias significativas a nivel de la presencia de un surco en el pectoral $\left(\mathrm{X}^{2} 1=20.38, \mathrm{p} \leq 0.001\right)$, donde la frecuencia encontrada en los húmeros masculinos (13.6\%) es muy superior a la encontrada en la muestra femenina (1.2\%). El músculo pectoral es un aductor y rotador medial del brazo (Platzer, 1987) y la presencia de un surco en el punto de unión del pectoral mayor es normal en subadultos, pero rara en adultos, en los que suele rellenarse quedando únicamente una ligera depresión (Mann y Murphy, 1990), aunque esto no sucede en Wamba. De este modo, encontramos una mayor frecuencia de este carácter en los varones, con predominancia del lado derecho (17.8\%). Es un marcador que prácticamente no aparece en mujeres. También se encontraron diferencias sexuales en la presencia de aplastamiento de la tuberosidad menor $\left(\mathrm{X}^{2} 1=15.56, \mathrm{p} \leq 0.001\right)$ siendo la frecuencia en 
Tabla 2: Frecuencia de los marcadores de actividad física de la extremidad superior, por sexo y lado.

\begin{tabular}{|c|c|c|c|c|c|c|c|c|c|c|}
\hline & \multicolumn{4}{|c|}{ Masculino } & \multicolumn{4}{|c|}{ Femenino } & \multirow{2}{*}{\multicolumn{2}{|c|}{$\frac{\text { Ambos Sexos }}{\text { Total }}$}} \\
\hline & \multicolumn{2}{|c|}{ Derecho } & \multicolumn{2}{|c|}{ Izquierdo } & \multicolumn{2}{|c|}{ Derecho } & \multicolumn{2}{|c|}{ Izquierdo } & & \\
\hline & $\mathrm{n}$ & $\%$ & $\mathrm{n}$ & $\%$ & $\mathrm{n}$ & $\%$ & $\mathrm{n}$ & $\%$ & $\mathrm{n}$ & $\%$ \\
\hline \multicolumn{11}{|l|}{ Húmero } \\
\hline Aplast. troc. mayor & $23 / 171$ & 13.5 & $18 / 150$ & 12.0 & $11 / 80$ & 13.8 & $4 / 85$ & 4.7 & $56 / 486$ & 11.5 \\
\hline Aplast. troc. menor & $53 / 171$ & 31.0 & $41 / 153$ & 26.8 & $11 / 83$ & 13.3 & $11 / 85$ & 12.9 & $116 / 492$ & 23.6 \\
\hline Surco pectoral & $31 / 174$ & 17.8 & $14 / 157$ & 8.9 & $0 / 83$ & 0.0 & $2 / 87$ & 2.3 & $47 / 501$ & 9.4 \\
\hline Surco redondo & $12 / 174$ & 6.9 & $12 / 157$ & 7.6 & $4 / 83$ & 4.8 & $3 / 87$ & 3.4 & $31 / 501$ & 6.2 \\
\hline Exóst. epitróclea & $19 / 167$ & 11.4 & $12 / 152$ & 7.9 & $5 / 80$ & 6.3 & $4 / 84$ & 4.8 & $40 / 483$ & 8.3 \\
\hline Exóst. epicóndilo & $45 / 172$ & 26.2 & $20 / 155$ & 12.9 & $18 / 83$ & 21.7 & $10 / 83$ & 12.0 & $93 / 493$ & 18.9 \\
\hline \multicolumn{11}{|l|}{ Radio } \\
\hline Exóst. tub. bicipital & $17 / 46$ & 37.0 & $16 / 54$ & 29.6 & $2 / 29$ & 6.9 & $3 / 39$ & 7.7 & $38 / 168$ & 22.6 \\
\hline \multicolumn{11}{|l|}{ Cúbito } \\
\hline Cresta supinador & $34 / 56$ & 60.7 & $33 / 60$ & 55.0 & $15 / 23$ & 65.2 & $19 / 38$ & 50.0 & $101 / 177$ & 57.1 \\
\hline Exóst. olécranon & $13 / 54$ & 24.1 & $17 / 59$ & 28.8 & $6 / 23$ & 26.1 & $2 / 40$ & 5.0 & $38 / 176$ & 21.6 \\
\hline Cresta pronador & $44 / 58$ & 75.9 & $47 / 59$ & 79.7 & $21 / 24$ & 87.5 & $36 / 41$ & 87.8 & $148 / 182$ & 81.3 \\
\hline Surco pronador & $1 / 58$ & 1.7 & $6 / 59$ & 10.2 & $2 / 24$ & 8.3 & $5 / 41$ & 12.2 & $14 / 182$ & 7.7 \\
\hline
\end{tabular}

los húmeros masculinos (29\%) casi el doble que en los femeninos (15.1\%). En esta tuberosidad se inserta el músculo subescapular, que es un rotador medial del brazo (Platzer, 1987).

Aunque no hemos encontrado asimetrías ni diferencias sexuales en el resto de indicadores, éstos tienden a ser más frecuentes en el lado derecho de varones. En cuanto a la presencia de un surco en la unión del músculo redondo mayor, es un rasgo común en subadultos que suele remodelarse en adultos, pero puede acentuarse debido a un trauma agudo o crónico del hombro (Mann y Murphy, 1990). El redondo mayor es un rotador medial, aductor y produce retroversión del brazo (Platzer, 1987). Así, parece que la rotación medial y aducción del brazo es un fenómeno que se produce con mayor frecuencia en los húmeros masculinos. Las actividades que provocaron la aparición de entesopatías a estos niveles podrían estar relacionadas, por ejemplo, con el transporte de carga sobre la espalda sosteniéndola con los brazos.

La presencia de exóstosis en la epitróclea puede estar relacionada con la edad, o bien con la actividad física, ya que es el punto de origen del tendón del pronador redondo (Mann y Murphy, 1990), músculo que prona el antebrazo y flexiona la articulación del codo. La epitróclea también sirve de unión al flexor superficial de los dedos, de la palma, y del carpo (Platzer, 1987). En el epicóndilo tiene su origen el músculo ancóneo que ayuda al tríceps en la extensión del brazo, así como los extensores de los dedos y el músculo supinador. Según las frecuencias obtenidas de exóstosis en epitróclea y epicóndilo, encontramos un mayor predominio de la extensión y supinación del brazo (18.9\%) frente a movimientos de pronación y flexión (8.3\%).

En el radio no se encontraron asimetrías para la presencia de exóstosis en la tuberosidad bicipital, aunque es más frecuente en el lado derecho. Existe, sin embargo, un marcado dimorfismo sexual $\left(\mathrm{X}^{2} 1\right.$ $=15.15, \mathrm{p} \leq 0.001)$ con una frecuencia en los radios masculinos (30.5\%) cinco veces mayor que en los femeninos (5.9\%). Varios estudios (Dutour, 1986; Pálfi y Dutour, 1996) señalan que la presencia de este parámetro suele ser más frecuente en varones y en el lado derecho, resultado coincidente con el nuestro. En la tuberosidad bicipital del radio se inserta el músculo bíceps braquial. La aparición de esta exóstosis podría ser una consecuencia de una actividad preferencial del brazo derecho en varones. Puede estar relacionado con una actividad en la que el individuo deba mantener su codo flexionado contra algo resistente, como sucede al transportar grandes pesos o arrastrarlos con los codos doblados. El uso del arado también puede producir la realización de fuerza con los brazos flexionados para guiar las 
mulas o bueyes, y con los dedos flexionados para sujetar las cinchas.

Frecuentemente se ha encontrado asociado con la hipertrofia de la tuberosidad ulnar a nivel de la inserción del músculo braquial y con el desarrollo de la cresta del supinador. Debido a la existencia de numerosas explicaciones independientes de estos síndromes en la bibliografía médica y paleopatológica (Dutour, 1986; Kennedy, 1989; Stirland, 1991) podemos únicamente considerar que estas alteraciones son la consecuencia más probable de una actividad preferencial y crónica que afectaba mecánicamente a los codos en los varones, por la supinación e hiperflexión de los brazos.

En el cúbito (Tabla 2) se encontró asimetría en el desarrollo de la cresta del supinador $\left(\mathrm{X}^{2} 1=\right.$ $10.49, \mathrm{p} \leq 0.01)$ en la muestra masculina; siendo más frecuente en el lado derecho. Este resultado concuerda con el obtenido por Kennedy (1983). En este trabajo obtuvo una mayor frecuencia en poblaciones cazadoras-recolectoras que se sabe usaron armas arrojadizas (bolas, bumerán, lanzas) para cazar. El acto de arrojar algo lleva consigo el desplazamiento angular del antebrazo como resultado de la rotación medial del brazo a nivel del hombro, hiperextensión del brazo y cambios abruptos de supinación a pronación. También existe una serie de condiciones médicas, estudiadas en el mundo del deporte, que constituyen más síndromes por efecto de movimientos articulares crónicos y repetidos, que debidos a fuerzas de impacto súbito. De entre éstos, podemos destacar: edemas, bursitis, fibrosis, osteocondrosis (inflamación del hueso y del cartílago articular) y osteocondritis disecans (inflamación del hueso y del cartílago con el resultado de la fragmentación de partes del cartílago dentro de la articulación). La fuerza muscular es el factor más crítico en la actividad de lanzamiento y en la formación de una cresta prominente en el origen del supinador. Este músculo es una ancha lámina oblicua situada bajo el braquiradial. Tiene su origen en el epicóndilo lateral del húmero y la porción adyacente del cúbito a lo largo de la cresta del supinador. Se inserta en el tercio superior de la cara lateral del radio. El supinador actúa en solitario en una supinación lenta, sin resistencia, o en una supinación rápida cuando el codo está extendido. Requiere la asistencia del bíceps en la supinación durante un movimiento rápido con el brazo flexionado. En este caso nos encontramos con una frecuencia muy alta, $57.1 \%$, de desarrollo de la cresta del supinador, en una población agricultora, por lo que no creemos que dicha frecuencia pueda ser debida a la presencia de patologías o a una actividad tal y como describe Kennedy. Más bien nos inclinamos por otro tipo de actividades que puedan encuadrarse dentro del marco de la agricultura, como son el uso de la hoz al segar, por ejemplo. De ser esto cierto, aunque los resultados obtenidos por Kennedy en verdad se relacionen con las actividades por ella descritas, no pueden considerarse como excluyentes de actividades realizadas en pueblos agricultores y que desemboquen en el desarrollo de similares marcadores. Esta hipótesis estaría de acuerdo con las conclusiones de otros trabajos (Angel et al., 1987; Kelley y Angel, 1987; Rathbun, 1987; Slaus, 1994) donde se asoció el fuerte desarrollo de la cresta del supinador con actividades tales como cavar, cortar madera y con el transporte de grandes pesos.

También se encontró asimetría en la presencia de exóstosis en la inserción del olécranon $\left(\mathrm{X}^{2}{ }_{1}=\right.$ $5.86, \mathrm{p} \leq 0.05)$, siendo ésta más frecuente en el lado derecho de la muestra femenina. En cuanto al dimorfismo sexual, aunque todos los parámetros son más frecuentes en varones, sólo se encontraron diferencias estadísticas en la presencia de exóstosis en el olécranon $\left(X^{2} 1=4.58, p \leq 0.05\right)$. El tríceps braquial es el principal extensor del codo, favoreciendo velocidad más que fuerza. Tiene tres cabezas que se originan en la escápula, bajo la cavidad glenoidea, en la cara posterolateral de la porción media de la diáfisis humeral y en la superficie posterior del tercio inferior de la diáfisis del húmero. Se inserta por medio de un tendón común a las tres cabezas en el olécranon del cúbito. Es el antagonista del bíceps y del braquial y es ayudado por el anconeo en la extensión del antebrazo (Kennedy, 1983; Platzer, 1987). Además de la importancia que tiene al determinar el lado preferente de un individuo, este marcador de presión en el extremo superior del cúbito, junto con la cresta del supinador, indican una actividad específica braquial. Sin embargo, en este caso no podemos asociar las actividades realizadas por ambos músculos, ya que la cresta del supinador presenta una frecuencia muy superior a la de exóstosis en el olécranon y además, en el olécranon se encontró asimetría y dimorfismo sexual. De este modo, la actividad que provocó el desarrollo de la cresta supinadora estaría compartida por el general de la población, similar en ambos sexos, mientras que la actividad generadora de exóstosis en el olécranon sólo sería realizada por el $21.6 \%$ de la 
población, y donde además existiría un patrón diferencial en ambos sexos, puesto que es más frecuente en varones y además, en las mujeres afecta de forma prioritaria a uno de los lados, el derecho. Lo mismo sucedía con el aplastamiento de la tuberosidad mayor del húmero, aunque con una frecuencia menor, pudiendo existir una relación entre los movimientos descritos para la realización de una actividad común.

Respecto a la hipertrofia de la cresta del pronador cuadrado, no se encontró asimetría ni dimorfismo sexual pero la frecuencia supera el $80 \%$. Este músculo actúa junto al pronador redondo en la pronación del antebrazo. De este modo, en rasgos generales, los movimientos de pronación y supinación del antebrazo, especialmente el primero, serían muy comunes en esta población, sin distinción de lado o sexo, y probablemente tengan algo que ver con actividades generalizadas, crónicas y comunes a varones y mujeres.

Además se ha encontrado, en general, mayor frecuencia de los parámetros que afectan al antebrazo, respecto al brazo, y esto podría indicar que en las actividades relacionadas con la agricultura tales como por ejemplo, trillar, aventar el grano, sembrar, cavar, recolectar, etc., la fuerza ejercida se aplica principalmente en codo y muñeca, con menor participación del hombro.

Es importante señalar que se ha encontrado una concordancia en los resultados para cada inserción muscular. Uno de los problemas del estudio de los marcadores de estrés musculoesquelético, además de los desarrollados en la introducción, consiste en que habitualmente se analiza cada inserción muscular de forma independiente y después se intenta asociar con una actividad específica, cuando los músculos no actúan sólos, sino en grupos, por lo que son estos grupos los que se deberían intentar asociar con la realización de una actividad (Stirland, 1998).

En la Tabla 3 aparecen las frecuencias de los marcadores de actividad física en la extremidad inferior. Sólo se ha detectado asimetría en la presencia de exóstosis en la cresta anterior intertrocantérica de los fémures masculinos $\left(\mathrm{X}^{2}{ }_{1}=\right.$ 4.91, $\mathrm{p} \leq 0.05$ ), siendo más frecuente en el lado izquierdo.

La cresta intertrocantérica sirve de inserción al músculo cuadrado femoral, que es un potente rotador externo y aductor del muslo (Platzer, 1987). El hecho de que exista asimetría para el desarrollo de la inserción de este músculo puede estar indicando la realización de una actividad específica en una pequeña parte de la población, especialmente por parte de los varones.

El patrón de asimetría encontrado en Wamba está de acuerdo con el fenómeno de asimetría cruzada descrito en diferentes trabajos (Schultz, 1937; Serra y Queiroz, 1943; Hernández-Asensio, 1972; Ruff, 1987), de modo que en el miembro superior está más desarrollado el lado derecho y en la extremidad inferior el izquierdo. De este modo, la presencia de esta entesopatía nos podría estar indicando una actividad postural, necesaria para la realización de una actividad con el miembro superior, tal y como ocurre, por ejemplo, al arar. En cuanto al dimorfismo sexual, todos los marcadores mostraron diferencias significativas, siendo las frecuencias mayores en la muestra masculina excepto para el caso de las alteraciones encontradas en la región situada por encima de la cara posterior del cóndilo medial.

En el trocánter menor se inserta el músculo iliopsoas que resulta de la unión de los músculos psoas mayor e iliaco. El iliopsoas es el principal flexor del muslo, posibilitando la marcha $\mathrm{y}$ contribuyendo, además, a la flexión del tronco y a la rotación lateral de la cadera (Platzer, 1987). Estos movimientos podrían relacionarse con ciertas actividades diferenciales como son arar o sembrar.

En la parte superior del trocánter mayor se inserta el músculo glúteo medio, un abductor del muslo. El glúteo mayor se inserta en la tuberosidad glútea, que es la localización que presenta mayor frecuencia de exóstosis. Este músculo es un extensor y rotador externo de la articulación de la cadera y entra en acción al subir escaleras y al levantarse de un asiento (Platzer, 1987).

En el caso de Wamba el desarrollo del glúteo mayor podría estar relacionado con la locomoción por la oreografía del lugar, con terreno accidentado y marcado por la presencia de numerosas colinas. Este resultado estaría de acuerdo con el observado por otros autores (Lai y Lovell, 1992). Según las frecuencias obtenidas, los varones desarrollarían una mayor actividad marchadora que las mujeres, puede que en relación a la actividad ganadera y de pastoreo que se desarrollaba en Wamba, o también debido al desplazamiento hacia las tierras de labor, hacia los bosques en busca de madera, o caza, y hacia Valladolid, con la que seguramente se mantenía una importante relación comercial. Los principales aductores del muslo, pectíneo, aductor corto, aductor mínimo y aductor mayor, se insertan a lo largo de la línea áspera y una parte del aductor mayor se inserta a través de un tendón, en el tubérculo aductor del cóndilo medial. 
Tabla 3: Frecuencia de los marcadores de actividad física de la extremidad inferior, por sexo y lado.

\begin{tabular}{|c|c|c|c|c|c|c|c|c|c|c|}
\hline & \multicolumn{4}{|c|}{ Masculino } & \multicolumn{4}{|c|}{ Femenino } & \multirow{2}{*}{\multicolumn{2}{|c|}{$\begin{array}{c}\text { Ambos Sexos } \\
\text { Total }\end{array}$}} \\
\hline & \multicolumn{2}{|c|}{ Derecho } & \multicolumn{2}{|c|}{ Izquierdo } & \multicolumn{2}{|c|}{ Derecho } & \multicolumn{2}{|c|}{ Izquierdo } & & \\
\hline & $\mathrm{n}$ & $\%$ & $\mathrm{n}$ & $\%$ & $\mathrm{n}$ & $\%$ & $\mathrm{n}$ & $\%$ & $\mathrm{n}$ & $\%$ \\
\hline \multicolumn{11}{|l|}{ Fémur } \\
\hline Exóst. troc. mayor & $52 / 210$ & 24.8 & $53 / 188$ & 28.2 & $14 / 81$ & 17.3 & $15 / 86$ & 17.4 & $134 / 565$ & 23.7 \\
\hline Exóst. troc. menor & $82 / 224$ & 36.6 & $64 / 200$ & 32.0 & $13 / 87$ & 14.9 & $12 / 92$ & 13.0 & $171 / 603$ & 28.4 \\
\hline Exóst. tub. glútea & $103 / 229$ & 45.0 & $97 / 208$ & 46.6 & $36 / 96$ & 37.5 & $30 / 96$ & 31.3 & $266 / 629$ & 42.3 \\
\hline Exóst. cresta intertr. & $9 / 231$ & 3.9 & $19 / 210$ & 9.0 & $2 / 96$ & 2.1 & $1 / 96$ & 1.0 & $31 / 633$ & 4.9 \\
\hline Exóst. línea áspera & $67 / 231$ & 29.0 & $52 / 210$ & 24.8 & $9 / 96$ & 9.4 & $5 / 96$ & 5.2 & $133 / 633$ & 21.0 \\
\hline Exóst. cón. medial & $42 / 231$ & 18.2 & $38 / 206$ & 18.4 & $27 / 95$ & 28.4 & $27 / 93$ & 29.0 & $134 / 625$ & 21.4 \\
\hline \multicolumn{11}{|l|}{ Tibia } \\
\hline Exóst. tub. ant. & $38 / 168$ & 22.6 & $26 / 151$ & 17.3 & $7 / 103$ & 6.8 & $9 / 116$ & 7.8 & $80 / 538$ & 14.9 \\
\hline Exóst. línea sóleo & $13 / 168$ & 7.7 & $11 / 151$ & 7.3 & $0 / 103$ & 0.0 & $1 / 116$ & 0.9 & $25 / 538$ & 4.6 \\
\hline Exóst. inserc. flexor & $6 / 166$ & 3.6 & $5 / 151$ & 3.3 & $1 / 102$ & 1.0 & $1 / 116$ & 0.9 & $13 / 535$ & 2.4 \\
\hline
\end{tabular}

Son potentes aproximadores y participan activamente en el acto de cruzar las piernas ya que además son rotadores y extensores de la cadera (Platzer, 1987; Aiello y Dean, 1990). Los aductores son también músculos muy importantes en la realización de ciertas actividades, tales como la equitación (Kapandji, 1996 b).

Diversos estudios han encontrado hipertrofia en las inserciones de los glúteos medio (trocánter mayor) y máximo (por encima de la tuberosidad glútea), aductores mayor y corto (línea áspera), cuádriceps femoral y peptíneo (por encima de la tuberosidad glútea). También estaban bien marcadas las inserciones del aductor mayor (cóndilo medial) en la epífisis inferior del fémur. Todo ello se ha relacionado con montar a caballo (ver Pálfi y Dutour, 1996). No podemos relacionar directamente la presencia de estas entesopatías con una actividad específica tal y como es la equitación ya que no disponemos de la información histórica adecuada. Es probable que se realizara el transporte de personas y carga utilizando algún tipo de caballería o bueyes, pero nuestros resultados no parecen apoyar exactamente esa teoría, ya que las alteraciones a nivel del cóndilo medial se producen con mayor frecuencia en mujeres. Debemos acudir entonces a otras hipótesis que ayuden a explicar este resultado, quizá la mayor frecuencia de una postura arrodillada en las mujeres debido a la realización de ciertas actividades tales como lavar la ropa, provocase la aparición de este tipo de alteraciones, de forma simétrica en ambos lados. Esto estaría de acuerdo con el hecho de que también se ha descrito el desarrollo de un crecimiento óseo a este nivel como una "huella tibial" debida a la flexión de la rodilla (ver Kennedy, 1989).

En la tibia no se encontró ninguna asimetría. En general las tibias masculinas presentaron mayores frecuencias que las femeninas, y estas diferencias fueron significativas para la presencia de exóstosis en la tuberosidad anterior $\left(\mathrm{X}^{2}{ }_{1}=16.69, \mathrm{p} \leq 0.001\right)$, y en la línea del sóleo $\left(\mathrm{X}^{2} 1=14.64, \mathrm{p} \leq 0.001\right)$. La tuberosidad anterior es el punto de inserción del tendón del cuádriceps femoral, principal extensor de la rodilla, y en la línea del sóleo se insertan el sóleo, flexor plantar del pie, y el poplíteo, rotador de la rodilla. Todos estos parámetros podrían estar relacionados con la movilidad del individuo, y puede que también con el soporte de cargas o pesos, aunque según las frecuencias obtenidas, éstos fenómenos de movilidad y carga parece que afectaron más a nivel femoral que tibial.

El dimorfismo sexual observado en el miembro inferior puede relacionarse con diferencias en carga y/o actividad locomotora, que junto con las diferencias de forma que se encontraron al analizar los índices morfológicos, podrían estar indicando la existencia de una actividad física diferencial en ambos sexos y/o más prolongada en los varones. Sin embargo, no podemos dejar de considerar que estas diferencias sexuales se deban a una diferente estructura de edad entre ambas subpoblaciones. Si la media de edad de los varones es superior a la de las mujeres, es más probable que desarrollen mayores frecuencias de estos marcadores de actividad física. Sin embargo, aunque así fuera hay 
otros factores a considerar, como son que una distribución de edades de este tipo no puede explicar el porqué algunos parámetros son más frecuentes en las mujeres, ni el porqué hay asimetría.

\section{Conclusiones}

En el análisis de los marcadores de actividad en la extremidad superior se encontró un mayor grado de aplastamiento de la tuberosidad mayor del húmero y una mayor presencia de exóstosis en el olécranon del cúbito en el lado derecho de las mujeres. Ambos caracteres pueden estar vinculados con un soporte asimétrico de la carga durante una actividad habitual y repetida.

La presencia de un surco en la inserción del músculo pectoral y el aplastamiento de la tuberosidad menor del húmero, la exóstosis en la tuberosidad bicipital del radio y la exóstosis en el olécranon del cúbito, resultaron estadísticamente más frecuentes en los varones, apuntando hacia actividades físicas que supusieran mayor esfuerzo de las articulaciones del codo y de la muñeca y menor participación del hombro. Estos resultados están de acuerdo con muchas de las tareas relacionadas con el proceso agrícola, tales como trillar, aventar el grano, sembrar, recolectar, etc. En la extremidad inferior únicamente se encontró asimetría para la presencia de exóstosis en la línea intertrocantérica del fémur a favor del lado izquierdo de varones.

El resto de marcadores muestran una mayor frecuencia en los varones y apuntan, además del soporte de grandes cargas o pesos, por un lado hacia la flexión del tronco y rotación lateral de la cadera, movimientos que podrían asociarse a ciertas actividades como arar, segar o sembrar; y por otro lado hacia una mayor actividad locomotora en dicho sexo, puede que en relación al desplazamiento hacia las tierras de labor, hacia el bosque para buscar leña, y hacia los pueblos de los alrededores y Valladolid, con fines comerciales.

Además se observa un mayor número de asimetrías en el miembro superior respecto al inferior. Este resultado concuerda con lo observado en otros trabajos (Ruff y Jones, 1981). La asimetría del miembro superior puede explicarse por la realización de actividades que requieran el uso prioritario de un lado, en este caso el derecho, que coincide con el lado predominante que aparece con mayor frecuencia en los distintos estudios realizados (Ruff y Jones, 1981).

Las asimetrías se han encontrado tanto en varones como en mujeres, afectando a marcadores diferentes. Este resultado parece apuntar a diferencias de actividad física entre ambos sexos, quizá debido a la participación de los varones en tareas que requirieran mayor fortaleza física, tales como el transporte de grandes pesos, labrar la tierra, arar o segar. En relación al dimorfismo sexual, éste es muy superior en el miembro inferior, donde afecta al $88.9 \%$ de los parámetros analizados, mientras que en la extremidad superior este porcentaje es del $36.4 \%$. El hecho de que exista un menor dimorfismo sexual en la extremidad superior se podría explicar por la realización de actividades hasta cierto punto parecidas entre ambos sexos, $\mathrm{o}$ al menos, tareas que aunque fueran diferentes dejaron unas marcas musculares similares.

\section{Bibliografía}

Aiello L. y Dean C. (1990) An introduction to human evolutionary anatomy. Academic Press. EEUU.

Angel JL., Kelley JO., Parrington M. y Pinter S. (1987) Life stresses of the free black community as represented by the first African Baptist Church, Philadelphia, 1823 - 1841. Am. J. Phys. Anthrop. 74: 213 - 229.

Churchill SE. y Morris AG. (1998) Muscle marking morphology and labour intensity in Prehistoric Khoisan foragers. Int. J. Osteoarch. 8(5): 390 - 411.

Dutour O. (1986) Enthesopathies (lesions of muscular insertions) as indicators of the activities of Neolithic Saharan populations. Am. J. Phys. Anthrop. 71: 221 $-224$.

Hernández-Asensio M. (1972) Análisis estadístico de algunas asimetrías óseas. Memoria de Licenciatura. UCM. Madrid.

Iscan MY. (1989) Age markers in the human skeleton. Charles C. Thomas Publisher. EEUU.

Kapandji IA. (1996 a) Cuadernos de fisiología articular. Cuaderno I. Miembro superior. $4^{\text {a }}$ edición. Masson. Francia.

Kapandji IA. (1996 b) Cuadernos de fisiología articular. Cuaderno II. Miembro inferior. $4^{\mathrm{a}}$ edición. Masson. Francia.

Kelley JO. y Angel JL. (1987) Life stresses of slavery. Am. J. Phys. Anthrop. 74: 199 - 211.

Kennedy KAR. (1983) Morphological variations in ulnar supinator crests and fossae as identifying markers of occupational stress. J. For. Sciences, 28 (4): $871-876$.

Kennedy KAR. (1989) Skeletal markers of occupational stress. En: Reconstruction of life from the skeleton. Eds. Iscan MY. y Kennedy KA. Alan R. Liss. EEUU.

Lai P. y Lovell NC. (1992) Skeletal markers of occupational stress in the fur trade: a case study from the Hudson's Bay Company Fur Trade Post. Int. J. 
Osteoarch. 2: 221 - 234.

Mann RW. y Murphy SP. (1990) Regional atlas of bone disease. A guide to paleopathologic and normal variation in the human skeleton. Charles C Thomas Publisher. Springfield, Illinois. EEUU.

Martín JC. (1986) El campesinado de la Cuenca del Duero (s. XIII-XV). Junta de Castilla y León. Valladolid.

Pálfi GY. y Dutour O. (1996) Activity-induced skeletal markers in historical anthropological material. Int. J. Anthrop. 11 (1): 41 - 55.

Pérez-Pérez A., Jiménez S. y Trancho GJ. (1991) Análisis de oligoelementos: estudio de la dieta en poblaciones de la Península Ibérica. En: Nuevas perspectivas en Antropología. Eds. Botella M., Du Souich Ph. y Jiménez S. Universidad de Granada. Granada.

Platzer W. (1987) Atlas de anatomía. Tomo I. Aparato locomotor. Ed. Omega. Barcelona.

Rathbun TA. (1987) Health and disease at a South Carolina plantation: 1840-1870. Am. J. Phys. Anthrop. 74: 239 - 253.

Robb J. (1994) Skeletal signs of activity in the Italian metal ages: methodological and interpretative notes. Hum. Evol. 9 (3): 215 - 229.

Robb J. (1998) The interpretation of skeletal muscle sites: a statistical approach. Int. J. Osteoarch. 8(5): 363 - 377.

Robledo B. (1993) Dimorfismo sexual del hueso coxal en una población española (s. XV-XVII). Una aplicación antropológica en Arqueología. Memoria de Licenciatura. Universidad de Granada. Granada.

Rogers J. y Waldron T. (1990) The paleopathology of enthesopathy. Ann. Rheum. Dis. 49: 169 - 172.

Rucquoi A. (1987) Valladolid en la Edad Media. 2 volúmenes. Junta de Castilla y León. Consejería de Educación y Cultura. Valladolid.

Ruff CB. (1987) Sexual dimorphism in human lower limb bone structure: relationship to subsistence strategy and sexual division of labor. J. Hum. Evol. 16: 391 - 416.

Ruff CB. y Jones HH. (1981) Bilateral asymmetry in cortical bone of the humerus and tibia - sex and age factors. Hum. Biol. 53 (1): 69 - 86.

Schultz AH. (1937) Proportions, variability and assymmetries of the long bones of the limbs and the clavicles in man and apes. Hum. Biol. 9 (3).

Serra JA. y Queiroz A. (1943) As proporções e assimetrias dos membres nos Portugueses. Contrib. para o Est. do Antrop. Portuguesa, VI.

Shaibani A., Workman R. y Rothschild BM. (1993) The significance of enthesopathy as a skeletal phenomenon. Clinical and Experimental Rheumatology, 11: 399 - 403.

Slaus M. (1994) Osteological evidence for peri-mortem trauma and ocupational stress in two medieval skeletons from Croacia. Coll. Anthrop. 18 (1): 165 - 175.

Steen SL. y Lane RW. (1998) Evaluation of habitual activities among two Alaskan Eskimo populations based on musculoskeletal stress markers. Int. J. Osteoarch. 8(5): 341 - 353.

Stirland AJ. (1991) Diagnosis of occupationally related paleopathology: Can it be done? En: Human paleopathology: current syntheses and future options. Eds. Ortner DJ. y Aufderheide AC. Smithsonian Institution Press. Washington.

Stirland AJ. (1993) Asymmetry and activity related changes in the male humerus. Int. J. Osteoarch. 3: $105-113$.

Stirland AJ. (1998) Musculoskeletal evidence for activity: problems of evaluation. Int. J. Osteoarch. 8(5): 354 - 362 .

Trancho GJ., Botella M. y Hernández M. (1991) Cribra orbitalia: incidencia y distribución en diferentes poblaciones de la Península Ibérica. En: Nuevas perspectivas en Antropología. Eds. Botella M., Du Souich Ph. y Jiménez S. Universidad de Granada. Granada.

Walker PL. (1995) Problems of preservation and sexism in sexing: some lessons from historical collections for paleodemographers. En: Grave reflections, portraying the past through cemeteries studies. Eds. Saunders R. y Herring A. Canadian Scholars' Press. Toronto. 